\title{
Sub-Poissonian statistics in order-to-chaos transition
}

\author{
Gagik Yu. Kryuchkyan ${ }^{1,2}$ and Suren B. Manvelyan ${ }^{2}$ \\ ${ }^{1}$ Yerevan State University, Manookyan 1, Yerevan, 375049, Armenia \\ and \\ ${ }^{2}$ Institute for Physical Research, National Academy of Sciences, \\ Ashtarak-2, 378410, Armenia
}

\begin{abstract}
We study the phenomena at the overlap of quantum chaos and nonclassical statistics for the time-dependent model of nonlinear oscillator. It is shown in the framework of Mandel Q-parameter and Wigner function that the statistics of oscillatory excitation number is drastically changed in order-to chaos transition. The essential improvement of sub-Poissonian statistics in comparison with an analogous one for the standard model of driven anharmonic oscillator is observed for the regular operational regime. It is shown that in the chaotic regime the system exhibits the range of suband super-Poissonian statistics which alternate one to other depending on time intervals. Unusual dependence of the variance of oscillatory number on the external noise level for the chaotic dynamics is observed. The scaling invariance of the quantum statistics is demonstrated and its relation to dissipation and decoherence is studied.
\end{abstract}

\section{INTRODUCTION}

The subject of this article is at the boundary of two basic phenomena in quantum physics nowadays attracting significant interest of a broad readership. One of them is quantum chaos, which has been intensively investigated in last two decades and has been found in many nonlinear systems. Quantum effects inherent to the chaotic behavior of classical systems are an interesting field pertaining to many problems of fundamental interest [1]. Another phenomenon is the generation of nonclassical states, including nonclassical states of light with a range of unique properties, e.g. sub-Poissonian statistics and squeezing. At present the problem of nonclassical states generation draws a lot of attention, both from the standpoints of pure knowledge and possible applications [2]. The principal purpose of the present paper is to study the interesting crossovers between these phenomena for an open time-dependent quantum system.

The majority of studies of quantum chaos for isolated or so called Hamiltonian systems, the classical counterparts of which are chaotic, focus on static properties such as spectral statistics of energy levels and transition probabilities between eigenstates of the system. A variety of studies have also been carried out to understand the features of time-dependent chaotic systems. By contrast to that, very little work has been done to investigate the quantum chaos for open nonlinear systems. The beginning of study of an open chaotic system can be dated back to the papers [3], where the authors have analyzed the kicked rotor and similar systems with discrete time interacting with a heat bath. Quite generally, chaos in classical conservative and dissipative systems with noise has completely different properties, e.g., strange attractors can appear only in dissipative systems. Noise can induce a number of interesting phenomena in transition from regular to chaotic behavior. In particular, it was shown that under definite conditions noise can induce chaos as well as destroy chaotic behavior (see for example [4]). In recent years much effort has been expended, both theoretically and experimentally, to explore the role of quantum fluctuations and noise in order-to-chaos transition for open systems. It is obvious, that the investigations in this area are connected with the quantum-classical correspondence problem in general and with environment induced decoherence and dissipation in particular. Recently this topic has been the focus of theoretical investigations. As a part of these studies it has been recognized [5] that the decoherence has rather unique properties for systems classical analogues of which are chaotic. In particular, the formation of sub-Plank structure in phase space has been discussed for chaotic system [6]. The connection between quantum and classical treatments of chaos was also realized by means of comparison between strange attractors on the classical Poincaré section and the contour plots of the Wigner functions [7]. Some studies have explored the connection between quantum dynamical manifestation of chaos and quantum entanglement which is possibly the most typical property of composite quantum systems [8]. From the experimental viewpoint, observation of dissipative effects and environment induced decoherence of dynamically localized states in the quantum delta-kicked rotor is carried out with the gas of ultracold caesium atoms in a magneto-optical trap subjected to a pulsed standing wave [9], [10]. Recently, new problems of chaotic motion have been studied in the experimental scheme with ultra-cold atoms in magneto-optical double-well potential [11]. Fluctuation and decoherence in three-level model of chaos-assisted tunneling have been experimentally studied for samples of caesium atoms in an amplitude-modulated standing wave of light [12].

The other problem of interest in this area relates to the production of various nonclassical states in systems with 
chaotic dynamics. Generation of non-classical states of light has been the subject of an intense theoretical and experimental activity since the first observations of squeezed states of light. Generation of squeezed states in chaotic systems was studied numerically and analytically by using a specific form of perturbation theory for the model of quantum kicked rotator in Refs. [13]. The result of numerical experiments on quadrature squeezing in simple quantum models that allow the transition to chaos in the classical limit are presented in [14]. In a recent paper sub-Poissonian statistics of oscillatory excitations numbers is established for chaotic dynamics of nonlinear oscillator [15]. The study of these phenomena provides a fundamental understanding of quantum fluctuations in quantum chaos and opens a way for new experimental studies of the quantum dissipative chaos in the field of Quantum Optics.

In addition to these important developments in investigations of quantum fluctuations and noise assisted to chaotic motion, in the present paper we examine mainly quantum statistics of elementary excitations for the model of nonlinear dissipative oscillator which shows the order-to-chaos transition. Our goal is twofold: first we study in detail what kind of statistics of oscillatory number states takes place in the quantum chaos, for both cases of vacuum- and temperature-reservoir, and second we analyze Wigner functions in a chaotic regime and strange attractors from the point of view of nonclassical statistics. It should be noted that sub-Poissonian statistics of oscillatory excitation numbers was established for chaotic dissipative dynamics and for the case of vacuum-reservoir in our previous paper [15]. In the part of the present paper we essentially expand this study particularly considering the important details as well as the case of coupling of the nonlinear oscillator with nonzero temperature reservoir. The other part of the paper is devoted to the problems of generation of sub-Poissonian light, parameter scaling in chaotic dynamics and to the external noise-induced effects.

The requirement in realization of this study is to have a proper quantum model showing both regular and chaotic dynamics in the classical limit. We propose a nonlinear oscillator driven by two forces at different frequencies for this goal. This model was proposed to study the quantum chaos in one of the our previous papers [7], where it was shown that the model is apt to verification in experiments. This model also is attractive for the following reasons: (i) it is different from a single driven nonlinear oscillator and kicked rotor, where a pulsed pump field could be used for realization of a chaotic regime, and may be proposed for experimental studies of quantum chaos in the area of quantum optics with a cw laser; (ii) dynamics of this model exhibits a rich phase-space structure including regimes of regular and chaotic motion depending on the system's parameters as well as displays remarkable quantum properties, in particular - nonclassical sub-Poissonian statistics of elementary excitations.

Open quantum systems are usually studied in the framework of reduced density matrix obtained by tracing over the degrees of freedom of environment. There have been suggestions (see, for example, [16]) that chaotic dissipative dynamics should be described on individual quantum trajectories in the framework of quantum state diffusion method (QSD) [17]. In addition to these studies, here we describe the quantum dissipative chaos using a statistical ensemble of trajectories which is usually realized in nature. Such description allows us to consider quantum manifestation of chaos in measurable quantities.

The plan of this paper is as follows. In Sec.II nonlinear oscillator driven by two forces at different frequencies is described and relevant regular and chaotic operational regimes are discussed. In Sec. III the formation of sub-Poissonian statistics of oscillatory excitations number for regular dynamics is analyzed and application to the generation of strong sub-Poissonian light is considered. The important novelty here is that the radial squeezing effect is much stronger, than an analogous one for the model of a single driven anharmonic oscillator. In Sec. IV we identify the kind of statistics of oscillatory number states which takes place for quantum chaos. Our central result here is that nonclassical, sub-Poissonian statistics can be realized for chaotic dynamics of the system under consideration. We also discuss the role of the dissipation in scaling relationships for the regular and chaotic motion as well as the dependence of results on the level of external noise. We summarize our results in Sec. V.

\section{ORDER TO CHAOS TRANSITION IN DOUBLY DRIVEN NONLINEAR OSCILLATOR}

In this section we briefly review the results on the model [7]. We treat dissipation and decoherence microscopically using a master equation which is solved numerically in the framework of QSD approach. This model is a nonlinear oscillator driven by two periodic monochromatic forces at different frequencies $\omega_{1}$ and $\omega_{2}$ for which the evolution of the reduced density operator $\rho$ is governed by the master equation

$$
\frac{\partial \rho}{\partial t}=-\frac{i}{\hbar}\left[H_{0}+H_{i n t,} \rho\right]+\sum_{i=1,2}\left(L_{i} \rho L_{i}^{+}-\frac{1}{2} L_{i}^{+} L_{i} \rho-\frac{1}{2} \rho L_{i}^{+} L_{i}\right) .
$$

We use the interaction picture corresponding to the transformation $\rho \rightarrow e^{-i \omega_{1} a^{+} a t} \rho e^{i \omega_{1} a^{+} a t}$ and the Hamiltonians are: 


$$
\begin{aligned}
H_{0} & =\hbar \Delta a^{+} a \\
H_{\text {int }} & =\hbar\left[\left(\Omega_{1}+\Omega_{2} \exp (-i \delta t)\right) a^{+}+\left(\Omega_{1}^{*}+\Omega_{2}^{*} \exp (i \delta t)\right) a\right]+\hbar \chi\left(a^{+} a\right)^{2} .
\end{aligned}
$$

Here $\Delta=\omega_{0}-\omega_{1}$ is the detuning, and $\delta=\omega_{2}-\omega_{1}$ is the difference between driving frequencies, which works as modulation frequency. $a, a^{+}$are boson annihilation and creation operators and $L_{i}$ are the Lindblad operators:

$$
L_{1}=\sqrt{(N+1) \gamma} a, L_{2}=\sqrt{N \gamma} a^{+},
$$

where $\gamma$ is the spontaneous decay rate of the dissipation process, and $N$ denotes the mean number of quanta of a heat bath. The couplings with two driving forces are given by Rabi frequencies $\Omega_{1}$ and $\Omega_{2}$, and $\chi$ is the strength of anharmonicity. The last terms in Eq. (1) concern the influence of the environment induced diffusion. We have followed the standard approach [18], [19] to dissipative quantum dynamics in the range of weak coupling of the system with the reservoir under the condition: $\gamma \ll k_{B} T / \hbar$, where $k_{B} T$ is the Boltzman's constant times temperature. Equation (1) is obtained in both the rotating wave and Markov approximations, without regard to the driving-induced noise effects [20].

To study the pure quantum effects we focus on the cases of very low reservoir temperatures which, however, ought to be still larger than the characteristic temperature $T \gg T_{c h}=\hbar \gamma / k_{B}$. This restriction implies that dissipative effects can be described selfconsistently in the frame of the Lindblad equation (1). Note here that for $N \ll 1$ and, even for vacuum reservoir, for $N \simeq 0$ the above restriction is valid for the majority of problems of quantum optics.

For $\Omega_{2}=0$ this equation describes the single driven, dissipative anharmonic oscillator, which is a well-known and archetypal model in nonlinear physics [21]. In case of doubly driven oscillator $\left(\Omega_{2} \neq 0\right)$, the interaction Hamiltonian (2) is explicitly time-dependent and the system exhibits regions of regular and chaotic motion. It should be noted that this model corresponds to a modified model of Duffing oscillator, i.e. an oscillator where the oscillatory frequency depends on the amplitude. To illustrate it, we first pay attention to the classical description.

\section{A. Classical equation and connection to the Duffing oscillator}

In the classical limit, the corresponding equation of motion for the dimensionless amplitude $\alpha(t)=\langle a(t)\rangle=$ $\operatorname{Tr}(a \rho(t))$ has the form

$$
\frac{d}{d t} \alpha=-\frac{1}{2} \gamma \alpha-i\left(\Delta+\chi\left(1+2|\alpha|^{2}\right)\right) \alpha-i\left(\Omega_{1}+\Omega_{2} \exp (-i \delta t)\right) .
$$

It is easy to demonstrate that this equation is the rotating-wave approximation of the equation for Duffing oscillator driven by two periodic forces. Indeed, let us start with the following equation for a time-dependent real amplitude

$$
\ddot{E}+\gamma \dot{E}+\omega_{0}^{2}\left[1+\frac{2 \chi}{\omega_{0}}\left(1+E^{2} / 2\right)\right] E=4 \omega_{0}\left(\Omega_{1} \cos \omega_{1} t+\Omega_{2} \cos \omega_{2} t\right) .
$$

which describes such oscillator. This equation is a generalization of the well-known Duffing oscillator to the case of two driving forces at different frequencies $\omega_{1}$ and $\omega_{2}$. To perform the rotating-wave approximation of the equation (5) we introduce the dimensionless complex amplitude $\alpha(t)$ as

$$
E(t)=\alpha(t) \exp \left(-i \omega_{1} t\right)+c . c .
$$

Neglecting also the second-order derivative of the slowly varying amplitude $\alpha(t)$ and using the inequality $\gamma|\dot{\alpha}| \ll$ $\omega_{0}^{2}|\alpha|$ one obtains (4).

\section{B. Operational regimes and measurable quantities}

The dynamics of doubly driven anharmonic oscillator exhibits a rich phase-space structure, including regimes of regular and chaotic motion, with two Rabi frequencies $\Omega_{1}$ and $\Omega_{2}$, and the difference $\delta$ between driving frequencies and detuning $\Delta$ being the control parameters. In study of the order-to-chaos transition in classical systems a useful tool is the examination of a constant phase map in the phase-space. In this context, our numerical analysis of the classical equation of motion in the $(X, Y)$ plane $(X=\operatorname{Re} \alpha, Y=\operatorname{Im} \alpha)$ shows that the classical dynamics of the system is regular in domains of small and large values of modulation frequency, i.e. $\delta \ll \gamma$ and $\delta \gg \gamma$, and also when one of the perturbation forces is much greater than the other: $\Omega_{1} \ll \Omega_{2}$ or $\Omega_{2} \ll \Omega_{1}$. The dynamics is chaotic in the 
range of parameters $\delta \gtrsim \gamma$ and $\Omega_{1} \simeq \Omega_{2}$, where the classical results for $X=\operatorname{Re} \alpha, Y=\operatorname{Im} \alpha$ obtained from Eq.(4) show that the classical strange attractors for Poincaré section are realized [7]. Thus, two ways of producing chaos in a controlled manner can be considered by monitoring the system through varying either the strength of the driving force, or the difference frequency $\delta$.

We will examine macroscopic quantum effects contributing to the chaotic behavior by considering both the Mandel $Q$ parameter [22] which describes the deviation of excitation number uncertainty from the Poissonian variance, i.

e. $Q=\left(\left\langle(\Delta n)^{2}\right\rangle-\langle n\rangle\right) /\langle n\rangle,\left\langle(\Delta n)^{2}\right\rangle=\left\langle\left(a^{+} a\right)^{2}\right\rangle-\left\langle a^{+} a\right\rangle^{2}$, and the Wigner function. Thus, we suggest that the distinction between regular and chaotic dynamics can be most easily understood by studying the dynamics of essentially quantum properties. In fact, our numerical analysis has shown that the quantum dynamical manifestation of chaotic behavior does not appear on ensemble averaged oscillatory excitation numbers, but is clearly seen on the probability distributions [7]. The connection between quantum and classical treatments of chaos can be realized by means of comparison between strange attractors in the classical Poincaré section, and the contour plots of the Wigner functions. Indeed, it was demonstrated in details [7] that for comparatively small values of the ratio $\chi / \gamma \lesssim 0.1$ the contour plots of Wigner functions are relatively close to the strange attractors. Such likeness of quantum and classical distribution vanishes in the deep quantum regime.

\section{SUB-POISSONIAN STATISTICS FOR REGULAR DYNAMICS}

Our numerical analysis of quantum effects assisting to chaotic dynamics is based on QSD approach that represents the reduced density operator averaging over the projectors onto the stochastic states $\left|\Psi_{\xi}\right\rangle$ of the ensemble: $\rho(t)=$ $M\left(\left|\Psi_{\xi}\right\rangle\left\langle\Psi_{\xi}\right|\right)$, where $M$ denotes the ensemble averaging. The corresponding equation of motion is [17]

$$
\begin{aligned}
\left|d \Psi_{\xi}\right\rangle= & -\frac{i}{\hbar} H\left|\Psi_{\xi}\right\rangle d t \\
& -\frac{1}{2} \sum_{i=1,2}\left(L_{i}^{+} L_{i}-2\left\langle L_{i}^{+}\right\rangle L_{i}+\left\langle L_{i}\right\rangle\left\langle L_{i}^{+}\right\rangle\right)\left|\Psi_{\xi}\right\rangle d t+\sum_{i=1,2}\left(L_{i}-\left\langle L_{i}\right\rangle\right)\left|\Psi_{\xi}\right\rangle d \xi_{i},
\end{aligned}
$$

where $\xi$ indicates the dependence on the stochastic process, the complex Wiener variables $d \xi_{i}$ satisfy the fundamental properties $M\left(d \xi_{i}\right)=0, M\left(d \xi_{i} d \xi_{j}\right)=0, M\left(d \xi_{i} d \xi_{j}^{*}\right)=\delta_{i j} d t$, and the expectation value $\left\langle L_{i}\right\rangle=\left\langle\Psi_{\xi}\left|L_{i}\right| \Psi_{\xi}\right\rangle$. For the oscillatory mean excitations number and the variance this method gives:

$$
\begin{gathered}
\langle n\rangle=\lim _{m \rightarrow \infty} \frac{1}{m} \sum_{\xi}^{m}\left\langle\Psi_{\xi}\left|a^{+} a\right| \Psi_{\xi}\right\rangle, \\
\left\langle(\Delta n)^{2}\right\rangle=\lim _{m \rightarrow \infty} \frac{1}{m} \sum_{\xi}^{m}\left(\left\langle\Psi_{\xi}\left|\left(a^{+} a\right)^{2}\right| \Psi_{\xi}\right\rangle-\left|\left\langle\Psi_{\xi}\left|\left(a^{+} a\right)\right| \Psi_{\xi}\right\rangle\right|^{2}\right),
\end{gathered}
$$

while the Wigner function is calculated with the formulas

$$
W(r, \theta)=\sum_{m, n} \rho_{n m} W_{m n}(r, \theta),
$$

in terms of matrix elements $\rho_{n m}=M\left(\left\langle n \mid \Psi_{\xi}\right\rangle\left\langle\Psi_{\xi} \mid m\right\rangle\right)$ of density operator in the Fock state representation, where $(r, \theta)$ are the polar coordinates in the complex phase-space plane $X=r \cos \theta, Y=r \sin \theta$. The coefficients $W_{m n}(r, \theta)$ are Fourier transform of matrix elements of the Wigner characterization function [23]

$$
W_{m n}(r, \theta)=\left\{\begin{array}{l}
\frac{2}{\pi}(-1)^{n} \sqrt{\frac{n !}{m !}} e^{i(m-n) \theta}(2 r)^{m-n} e^{-2 r^{2}} L_{n}^{m-n}\left(4 r^{2}\right), m \geq n \\
\frac{2}{\pi}(-1)^{m} \sqrt{\frac{m !}{n !}} e^{i(m-n) \theta}(2 r)^{n-m} e^{-2 r^{2}} L_{m}^{n-m}\left(4 r^{2}\right), n \geq m
\end{array}\right\},
$$

where $L_{p}^{q}$ are the Laguerre polynomials. 


\section{A. Controlled strong sub-Poissonian statistics}

First we discuss the case of classically regular behavior of the considered model assuming the interaction of the system with vacuum reservoir, $N=0$. In our numerical analysis we focus, for simplicity, on the case of strong anharmonicity considering the parameters from $\chi / \gamma=0.05$ to $\chi / \gamma=0.7$. In the latter case the maximum mean number of oscillatory excitations $\langle n\rangle=15$, while in the former case it is equal to $\langle n\rangle=250$.

The results for time evolution of the mean oscillatory excitation number as well as the Mandel $Q$ parameter averaged over quantum trajectories, are depicted in Fig.1 and 2 for the parameters: $($ curve 1$) \chi / \gamma=0.7, \Delta / \gamma=-15$, $\Omega_{1} / \gamma=10.2, \Omega_{2} / \gamma=13.5$, and $\delta / \gamma=5 ;($ curve 2$) \chi / \gamma=0.05, \Delta / \gamma=-15, \Omega_{1} / \gamma=38.18, \Omega_{2} / \gamma=49.5$, and $\delta / \gamma=5$. As we see the $Q$ parameter shows a time-dependent modulation with a period $2 \pi / \delta$ and formation of sub-Poissonian statistics, $(Q<0)$ in which the oscillatory excitation number fluctuations are squeezed below the coherent level $\left\langle(\Delta n)^{2}\right\rangle\langle\langle n\rangle)$, for time intervals exceeding the transient time, $t \gamma \gtrsim 5$. The Mandel parameter reaches its minimum $Q_{\min } \simeq-0.9$ and maximum $Q_{\max } \simeq-0.38$ values for definite time intervals, for the case illustrated in Fig. 2 (curve 2). Moreover, the degree of sub-Poissonian statistics increases with increasing of mean oscillatory excitation numbers at definite time intervals. Below we demonstrate the formation of sub-Poissonian statistics by analyzing the Wigner function. Figs. 3 show the Wigner function and its contour-plot at the fixed moments of time $t_{k}=[7.13+(2 \pi / \delta) k] \gamma^{-1},(k=0,1,2, \ldots)$ exceeding transient time. We find that the Wigner function is located around the point $X=0, Y=-10$ and its contour-plot has narrow crescent form with the origin of phase space as its centrum. Another peculiarity is that the Wigner function is nonstationary. As calculations show, during the period of modulation $2 \pi / \delta$ it revolves around the origin of the phase-space. The radial squeezing that represents the known property of the anharmonic oscillator to produce the excitation number squeezing is also clearly seen in the figure. The important novelty here is that the radial squeezing effect in this model is much stronger, than an analogous one for the model of single driven anharmonic oscillator. Indeed, for $\Omega_{2}=0$, when we turn to the single driven anharmonic oscillator, the Mandel parameter is $Q=-0.72$ for the same parameters as in Fig.2 (curve 2).

\section{B. Application to the generation of nonclassical light}

This model can be proposed for the realization of optical schemes generating light with strong sub-Poissonian statistics. One of the candidates may be a scheme involving self-phase modulation in an optical cavity pumped by two coherent laser fields. In this scheme the anharmonicity of the oscillatory mode comes from photon-photon interactions in the $\chi^{(3)}$ medium inserted in the cavity, while dissipative effects arise from leakage of photons through the cavity mirrors, which damps the mode. In the case of a ring cavity the evolution of the mode at frequency $\omega_{0}$ is described by the Hamiltonian (2) with operators $a, a^{+}$being the annihilation and creation operators. The coupling constant $\chi$ of the Kerr interaction is proportional to the third-order nonlinear susceptibility $\chi^{(3)}$, and $\Omega_{1}$ and $\Omega_{2}$ are the Rabi frequencies corresponding to two classical coherent fields at the frequencies $\omega_{1}$ and $\omega_{2}$, respectively. The cavity damping and noise effect are described by the Lindblad operator (3), where $\gamma$ is the damping rate and $N$ is the mean number of thermal reservoir photons.

For the concrete scheme of light generation in a ring cavity, when the coupling of "in" and "out" fields occurs at the different mirrors, the cavity-output field operator $a_{\text {out }}=\sqrt{2 \gamma} a$, while the cavity-output intensity in photon number units per unit time is determined by $n_{\text {out }}=\left\langle a_{\text {out }}^{+} a_{\text {out }}\right\rangle=2 \gamma\left\langle a^{+} a\right\rangle$.

It is easy to verify that in this case the results depicted on the Fig.1 relate also to the normalized intensity $n_{\text {out }} / 2 \gamma$ of output light. For this scheme the measure of photocount statistics of detected output photons can also be directly expressed in terms of itracavity photons, namely Mandel factor $Q$. Indeed, in sub-Poissonian light experiments the observable quantity is the deviation of the variance of photocount number of output photons defined as $Q_{i}=$ $\left(\left\langle\left(\delta n_{i}\right)^{2}\right\rangle-\left\langle n_{i}\right\rangle\right) /\left\langle n_{i}\right\rangle$, where $\left\langle n_{i}\right\rangle$ is the mean number of photocounts [22]. We consider a short counting time $T \ll 2 \pi / \delta$ to be accurate in detection of the time-dependent output field modulated with period $2 \pi / \delta$. In this approximation $Q_{i}=\alpha T n_{\text {out }}\left(g_{\text {out }}^{(2)}-1\right)$, where $\alpha$ is a dimensionless quantum output of a detector, and $g_{\text {out }}^{(2)}=$ $\left\langle a_{\text {out }}^{+} a_{\text {out }}^{+} a_{\text {out }} a_{\text {out }}\right\rangle / n_{\text {out }}^{2}$ is the second order correlation function of the output field. Further, in terms of intracavity photon numbers $Q_{i}=2 \gamma \alpha T Q$, and hence the normalized quantity $Q_{i} / 2 \gamma \alpha T$ reduces to the Mandel parameter for intracavity photons. Thus, the results of Fig.2 are applicable to quantum statistics of light and indicate the timedependence of the normalized quantity $Q_{i} / 2 \gamma \alpha T$. In particular, the most strong sub-Poissonian statistics of generated light takes place for time intervals at which the output intensity reaches its maximal values.

It is natural to explain such strong sub-Poissonian statistics of light by a nontrivial interference effect or by the mutual interference of two coherent fields in nonlinear medium. However such analysis is difficult to perform because 
the system cannot be analyzed analytically in details. In this context we restrict ourselves by the remark that the system under consideration is an example of a quantum systems in contact with environment, where the controlling and ordering of dissipative dynamics as well as quantum statistics are realized through an external time-dependent force. Some examples have been proposed in Refs. [24].

\section{NONCLASSICAL STATISTICS FOR CHAOTIC DYNAMICS}

We now study the emergence of quantum chaos, which is expected to manifest itself as crucial changes in the above results in the classically chaotic operational regime. One of the ways to realize the controlling transition from regular to chaotic dynamics is to vary the strength $\Omega_{2}$ of the second force in the range from $\Omega_{2} \ll \Omega_{1}$ to $\Omega_{2} \gg \Omega_{1}$. In the limit $\Omega_{2} \ll \Omega_{1}$ the system is reduced effectively to the model of single driven anharmonic oscillator, which exhibits bistability for a definite range of parameters $\chi, \Delta, \Omega_{1}$, and $\gamma[21]$ On increasing the amplitude $\Omega_{2}$ the system transits from the regular to the chaotic regimes at $\Omega_{1} \simeq \Omega_{2}$. In the limit $\Omega_{2}>\Omega_{1}$ the regular dynamics is restored. The results of numerical simulation of the Mandel parameter in the regime of chaos are shown on Figs.4 for the parameters: (curve 1) $\chi / \gamma=0.7, \Delta / \gamma=-15, \Omega_{1} / \gamma=\Omega_{2} / \gamma=10.2$, and $\delta / \gamma=5 ;($ curve 2$) \chi / \gamma=0.05, \Delta / \gamma=-15$, $\Omega_{1} / \gamma=\Omega_{2} / \gamma=38.18$, and $\delta / \gamma=5$. Surprisingly, the excitation-number fluctuations are also squeezed below the coherent level for the considered chaotic regime. However, in contrast to the previous regular regime, the excitation number exhibits both sub-Poissonian $(Q<0)$ and super-Poissonian $(Q>0)$ statistics, that alternate in definite time intervals. The minimum and maximum values of $Q$ in time intervals during one modulation period are equal to $Q_{\min } \simeq-0.7$ and $Q_{\max } \simeq 0.6$ [for case (b)]. Thus, Figs.2 and 4 show the drastic difference between the behavior of Mandel parameter for regular and chaotic dynamics. It means that the variance of oscillatory number fluctuations may be used for testing of quantum chaos.

Now we illustrate the emergence of nonclassical sub-Poissonian statistics in the doubly driven nonlinear oscillator in its transition from regular to chaotic dynamics using the phase-space symmetry properties of the Wigner function. One can make sure of that by comparing the contour-plots of Wigner function for sub-Poissonian and super-Poissonian statistics. The results of ensemble-averaged numerical calculations of both the Wigner function and its contour-plot at fixed time intervals $t_{k}=[6.96+(2 \pi / \delta) k] \gamma^{-1},(k=0,1,2, \ldots)$ are shown in Figs. 5 (a, b) respectively. As we see the contour-plot for chaotic motion still has the radial squeezed form [see Fig. 5 (b)]. This result takes place for $t_{k}=[6.96+(2 \pi / \delta) k] \gamma^{-1},(k=0,1,2, \ldots)$ at which the Mandel parameter reaches its minimum value $Q_{\text {min }} \simeq-0.7$. In subsequent time intervals during the period of modulation the level of excitation number fluctuations increases, and as a result the radial squeezing in contour-plot decreases. This result is depicted in Figs. 6 (a) for the same parameters as in Fig.5(a), but for time-intervals $t_{k}=[7.325+(2 \pi / \delta) k] \gamma^{-1},(k=0,1,2, \ldots)$. The Poincaré section corresponding to these time moments are shown in Fig. 6 (b).

As we see there is a possibility to control the statistics of excitation numbers of the oscillator by means of variation of the field strengths $\Omega_{2}$. One can obtain the following regime of generation: purely sub-Poissonian statistics (Fig. 2 (curves 1,2)), oscillating between super- and sub-Poissonian (Fig. 4 (curves 1, 2)). Moreover, for certain values of the parameters we achieve an essential improvement of sub-Poissonian statistics in comparison with a single driven anharmonic oscillator, $\Omega_{2}=0$. In this context it seems important to analyze in more detail the Mandel parameter in order-to-chaos transition. To this end we have also studied the behavior of the $Q$ factor versus the controlling parameter $\Omega_{2} / \gamma$. The results of numerical calculations at a definite time moments exceeding the transient time, when the minimum of Mandel parameter was realized, are displayed in Fig.7. As is seen the result is displayed in the complicated form and shows the essential improvement of sub-Poissonian statistics in the chaos-to-order transition taking place for high values of $\Omega_{2} / \gamma$. The sub-Poissonian statistics goes bad for small values of Rabi frequency $\Omega_{2} / \gamma$ as well as for its intermediate values corresponding to the chaotic regime.

There is a particularly interesting feature to notice in the comparative analysis of strange attractors and contour plots of the Wigner function [Figs.5 (b,c) and Fig. 6 (a,b)]. We can conclude that it is possible to predict the shape of Wigner function by the knowledge of Poincaré section. Therefore, it is possible to predict the squeezing in quantum system by the knowledge of Poincaré section of its chaotic classical counterpart, that occurs by using Fig. 5 (c). This opens new possibilities to guess intuitively what kind of states will appear in quantum chaotic system by treating its classical chaotic map.

\section{A. Parameter scaling and dependence on external noise}

Further investigation of this model allows to establish other properties of dissipative quantum chaos. It is easy to verify that Eq.(4) is invariant with respect to the following scaling transformation of the complex amplitude $\alpha \rightarrow \alpha^{\prime}=$ 
$\lambda \alpha$, where $\lambda$ is a real positive dimensionless coefficient, if the parameters $\chi, \Delta, \Omega_{1}, \Omega_{2}$ are correspondingly transformed as: $\chi \rightarrow \chi^{\prime}=\chi / \lambda^{2}, \Delta \rightarrow \Delta^{\prime}=\Delta+\chi\left(1-1 / \lambda^{2}\right), \Omega_{1,2} \rightarrow \Omega_{1,2}^{\prime}=\lambda \Omega_{1,2}$. As we see the scaling transformations do not include the rate of the dissipative process $\gamma$ and hence can be written by using of dimensionless parameters as the follows: $\Delta / \gamma \rightarrow \Delta^{\prime} / \gamma^{\prime}=\Delta / \gamma+(\chi / \gamma)\left(1-1 / \lambda^{2}\right), \chi / \gamma \rightarrow \chi^{\prime} / \gamma^{\prime}=\chi / \gamma \lambda^{2}, \Omega_{1,2} / \gamma \rightarrow \Omega_{1,2}^{\prime} / \gamma^{\prime}=\lambda \Omega_{1,2} / \gamma$. This scaling property of the classical equation for the chaotic dynamics leads to the symmetry of strange attractors which, for definite different sets of parameters, have the same form in the phase-space and differ from each other only in scale. Some examples of such behavior have been presented in our previous paper [7]. So, we conclude that the parameter scaling happens for the classical trajectory dynamics for both regular and chaotic regimes. It is interesting to analyze such scaling from the point of view of quantum-statistical theory and its relevance for decoherence and dissipation. It should be noted, that recently several studies have been devoted to the scaling relationships in the decoherent quantum-classical transition for chaotic systems (see [25] and reference therein). The quantum-classical transition is now understood to be affected by the relative size of $\hbar$ for a given system as well as by the parameter $D$, a measure of decoherence of quantum system of interest. Further, in systems where the classical equation is chaotic, the transition is also affected by the chaos in the system, and thus by $\theta$, the Lyapunov exponent of the classical trajectory dynamics. Indeed, above mentioned study [25] was devoted to scaling relationships involving $\hbar, D, \theta$. Here we hold on another approach and present the results on scaling relationships, involving the system parameters $\chi / \gamma$, $\Delta / \gamma$, and $\Omega_{1,2} / \gamma$, in the framework of oscillatory numbers and its statistics. The goal is to analyze what kind of parameter scaling we can achieve in a quantum ensemble theory in the presence of quantum noise. We show below that such parameter scaling occurs for wider ranges of the parameters, but for not large values of the parameter $\chi / \gamma$. As our numerical calculations show $Q$ Mandel factor is approximately invariant under scaling transformations, i.e. $Q\left(\chi / \gamma, \Delta / \gamma, \Omega_{1,2} / \gamma\right) \simeq Q\left(\chi / \lambda^{2} \gamma, \Delta / \gamma+\chi / \gamma\left(1-1 / \lambda^{2}\right), \lambda \Omega_{1,2} / \gamma\right)$. This is illustrated in Fig.8 (a) for a time interval during the period of modulation and for the regular dynamics. Two sets of the parameters are: (curve 1) $\chi / \gamma=0.1$, $\Delta / \gamma=-15, \Omega_{1} / \gamma=27, \Omega_{2} / \gamma=35$, and $\delta / \gamma=5$; (curve 2) $\chi / \gamma=0.05, \Delta / \gamma=-15, \Omega_{1} / \gamma=38.18, \Omega_{2} / \gamma=49.5$, and $\delta / \gamma=5$ with scaling parameter $\lambda=\sqrt{2}$. As we see, the scaling invariance is valid within 0.02 . We also easily observe that the degree of violation of the scaling increases with increasing of the ratio $\chi / \gamma$ due to the moving of the system to a deeper quantum regime. See, in particular Fig. 2 and Fig. 4 where we have shown the results for two sets of parameters which are connected with a scaling transformation with $\lambda=\sqrt{14}$. Analogous results take place for the ranges of the parameters leading to chaotic dynamics. In Fig. 8(b) we illustrate the scaling of the $Q$ factor for the parameters: (curve 1) $\chi / \gamma=0.1, \Delta / \gamma=-15, \Omega_{1} / \gamma=\Omega_{2} / \gamma=27$, and $\delta / \gamma=5$; (curve 2) $\chi / \gamma=0.05, \Delta / \gamma=-15$, $\Omega_{1} / \gamma=\Omega_{2} / \gamma=38.18$, and $\delta / \gamma=5$ with scaling parameter $\lambda=\sqrt{2}$. The breaking of scaling invariance in deep quantum chaotic regime is shown in Fig. 4 with scaling parameter $\lambda=\sqrt{14}$. We emphasize that even though we have motivated the study of the parameter scaling with connection to dissipation and decoherence, the results obtained can be useful for other problems.

In the preceding sections we have studied statistics of excitation number in the pure quantum regime assuming coupling of nonlinear oscillator with a reservoir at zero temperature. In this part of the section we address the question of how external noise affects statistics of excitation numbers. We calculate the Mandel parameter with formulas (8), (9) as in the previous case of vacuum reservoir. Calculating quantum trajectories, we set the system initially to the vacuum oscillatory state and integrate Eq. (7) with Lindblad operators (3) for series of definite values of $N$. The results of numerical calculations at definite time moments exceeding the transient time are depicted in Figs.9, where minimum values of the $Q$ factor in time during the period $2 \pi / \delta$ versus external noise strength are presented. The curve (1) describes the case of a single driven anharmonic oscillator $\left(\Omega_{2}=0\right)$ for the same parameters as for the curve (2). As we see for the regular regime (curve(2)) $Q$ increases linearly with $N$. The Mandel parameter is $Q=-0.88$ for $T=0 \mathrm{~K}(N=0)$ ), but is $30 \%$ larger for $N=0.5$ (curve 2). An analogous linear dependence takes place for the single driven anharmonic oscillator [26]. However, for noisy chaotic regime the $Q$ factor is approximately independent of noise and even slightly decreases for comparatively large ranges of $N$ [Fig. 9 (curve 3)]. Such behavior can be explained by additional noise-induced mechanisms in chaotic evolution, namely, by chaos-to-order transition under influence of external noise.

\section{CONCLUSION}

Thus, in this work we have numerically studied the phenomena at the overlap of chaos, dissipation and nonclassical statistics for the time-dependent nonlinear model, namely Duffing oscillator driven by two periodic forces, showing order-to-chaos transition. We have shown that physical systems based on this model have a potential for generation of high-degree sub-Poissonian light as well as for observation of quantum-statistical effects that accompany chaotic dynamics. We have analyzed the mean oscillatory excitation number, Mandel $Q$ factor and the Wigner function for both regular and chaotic dynamics. We have shown in detail that the statistics of oscillatory excitation number 
is drastically changed in the order-to-chaos transition. Particularly interesting is the essential improvement of subPoissonian statistics in the regular operational regime of the scheme outlined above in comparison with an analogous one for the standard model of a single driven anharmonic oscillator. We have observed that in the chaotic regime the system exhibits ranges of different statistics depending on the modulation time, which alternate between suband super-Poissonian statistics. Another important conclusion concerns the conformity between strange attractors and contour plots of the Wigner function, that may be used for qualitative testing of quantum states by treating its classical chaotic map. Considering noise-induced effects we conclude that $Q$ parameter increases with increasing of the noise level $N$ for regular dynamics. This result is in good agreement with the results obtained for the single driven anharmonic oscillator [26]. However, $Q$ parameter is almost insensitive to the level of external noise for the case of chaos.

\section{Acknowledgments}

G. Yu. K. gratefully acknowledges useful discussions with H.K. Avetisian and support by the ISTC grant No. A-353. S. B. M. thanks Zh. Haroutunyan for technical support.

[1] F. Haake, "Quantum Chaos" (Springer Verlag, Berlin, 2001); "New Directions in Quantum Chaos", eds. by G. Casati et al. (IOS press, Amsterdam, 2002).

[2] Special Issue on Squeezed States of Light JOSA B, 4, No.10 (1987); Special Issue on Quantum Noise Reduction in Optical Systems, edited by E. Giacobino and C. Fabre, J. Appl. Phys. B 55, 216 (1992); L. Davidovich. Rev. Mod. Phys., 68, 127 (1996).

[3] E. Ott, M. Antonsen Jr, and J. P. Hanson, Phys. Rev. Lett. 53, 2187 (1984); T. Dittrich and R. Graham, Ann. Phys. (NY) 200, 363 (1990).

[4] J.B. Gao, S.K. Hwang, and J.M. Liu, Phys.Rev. Lett., 82, 1132 (1999).

[5] W. H. Zurek and J. P. Paz, Phys. Rev. Lett. 72, 2508 (1994); 75, 351 (1995); S. Habib, K. Shizume, and W. H. Zurek, ibid. 80, 4361 (1998); J. Gong and P. Brumer, Phys. Rev. E 60, 1643 (1999).

[6] W. H. Zurek, Nature, 412, 712 (2001).

[7] H. H. Adamyan, S. B. Manvelyan, and G. Yu. Kryuchkyan, Phys. Rev. E 64, 046219 (2001).

[8] K. Furuya, M.C. Nemes, and G.Q. Pellegrino, Phys. Rev. Lett. 80, 5524 (1998); A. Lakshminarayan, Phys. Rev. E 64, 036207 (2001).

[9] H. Ammann, et al., Phys. Rev. Lett. 80, 4111 (1998).

[10] B. G.Klappauf, et al., Phys. Rev. Lett., 81, 1203 (1998); 82, 241 (1999).

[11] D. J. Haycock, et al., Phys. Rev. Lett. 85, 3365 (2000); S. Ghose, M. Alsing, and I. Deutsch, quant-ph/010285.

[12] D.A. Stek, W.H. Oskay, and M.G. Raizen, Phys. Rev. Lett. 88, 120406 (2002).

[13] K.N. Alekseev, J. Perina, Phys. Lett., 231, 373 (1997); Phys. Rev. E, 57, 4023 (1998); K.N. Alekseev and D.S. Primak, quant-ph/0111044 (2002).

[14] R.-N. Xie and G. Xu, Phys. Rev. E 54, 1402 (1996); 54, 2132 (1996).

[15] G. Yu. Kryuchkyan, and S. B. Manvelyan, Phys. Rev. Lett., 88, 094101 (2002).

[16] T.P.Spiller and J.F.Ralph, Phys. Lett. A 194, 235 (1994)

[17] N. Gisin and I.C. Percival, J. Phys. A 25, 5677 (1992); A 26, 2233 (1993); A 26, 2245 (1993); I.C. Percival, "Quatnum State Diffusion", (Cambridge University Press, Cambridge, 2000).

[18] C.W. Gardiner, "Handbook of Stochastic Methods" (Berlin, Springer, 1986).

[19] U. Weiss,Quantum Dissipative Systems, Vol.2 of Series in Modern Condensed Matter Physics (World Scientific, Singapore, 1993).

[20] S. Kohler, T. Dittrich, and P. Hänggi, Phys. Rev. E 55, 300 (1997).

[21] P.D. Drummond and D. Walls, J. Phys. A 13725 (1980); D.J. Daniel and G.J. Milburn, Phys. Rev. A 39, 4628 (1989) ; V. Perinova and A. Luks, Phys. Rev. A 41, 414 (1990); D. Bortman and A. Ron, Opt. Commun. 108, 253 (1994); Phys. Rev. A 52, 3316 (1995); A. Bandyopadhyay and G. Gangopadhyay, Journ. Mod. Opt. 43, 487 (1996); E.M. Wright, T. Wong, M.J. Collett, S.M. Tan, and D.F. Walls, Phys. Rev. A 56, 591 (1997); K.V. Kheruntsyan, Quant. Semiclass.Opt. 1, 225 (1999).

[22] L. Mandel and E. Wolf, "Optical coherence and quantum optics", (Cambridge University Press, Cambridge, 1995).

[23] B.M. Garraway and P.L. Knight, Phys. Rev. A 49, 1266 (1994).

[24] F. Grossman, T. Dittrich, P. Jung, and P. Hänggi, Phys.Rev. Lett. 67, 516 (1991); M. Grifoni and P. Hänngi, Phys. Rep. 304, 219 (1998); L. Viola, E. Knill, and S. Lloyd, Phys. Rev. Lett. 82, 2414 (1999); D. Vitali and P. Tombesi, Phys. Rev. A 59, 4178 (1999); G.S. Agarwal, Phys. Rev. A 61, 013809 (2000).

[25] A.K. Pattanayak and B. Sundaram, quant-ph/0206069 (2002). 
[26] D. Enzer and G. Gabrielse, Phys. Rev. Lett. 78, 1211 (1997).

\section{FIGURE CAPTIONS}

Fig. 1. Excitation numbers in regular regime for two sets of parameters (see text). Excitation numbers for curve 1 is multiplied on 14. The averaging is over 2000 trajectories.

Fig. 2. The Mandel factor for the same as in Fig.1 parameters. The averaging is over 2000 trajectories.

Fig. 3. The Wigner function (a) and its contour-plot (b) for the regular regime averaged over 3000 trajectories.

Fig. 4. The Mandel factor in chaotic regime for two sets of parameters (see text).

Fig. 5. The Wigner function (a) and its contour-plot (b) averaged over 3000 trajectories. The Poincaré section (c) (approximately 20000 points) plotted at times of the constant phase, when the maximal sub-Poissonian statistics in quantum regime is reaslized. In both cases the dimensionless parameters are in the range of chaos:

Fig. 6. The contour-plot of Wigner function (a) and corresponding Poincaré section (b) (approximately 10000 points) constructed at time moments when the maximal super-Poissonian statistics is reaslized. The parameters and averagind for case (a) are the same as in Fig. 5.

Fig. 7. The behavior of the minimums of the Mandel factor $Q_{\min }$ versus the controlling parameter $\Omega_{2} / \gamma$. The parameters are: $\chi / \gamma=0.1, \Delta / \gamma=-15, \Omega_{1} / \gamma=27, \delta / \gamma=5$.

Fig. 8. The scaling of the $Q$ factor for two sets of parameters (see text) in regular (a) and chaotic (b) regimes. The averaging is over 1000 trajectories.

Fig. 9. The dependence of $Q_{\min }$ on the noise intensity for: (curve 1$)$ single driven nonlinear oscillator $(\chi / \gamma=0.1$, $\Delta / \gamma=-15, \Omega_{1} / \gamma=27$, and $\delta / \gamma=5$ ), (curve 2) doubly driven nonlinear oscillator in regular $\left(\Omega_{2} / \gamma=35\right)$, and (curve $3)$ in chaotic $\left(\Omega_{2} / \gamma=\Omega_{1} / \gamma=27\right)$ regimes. 


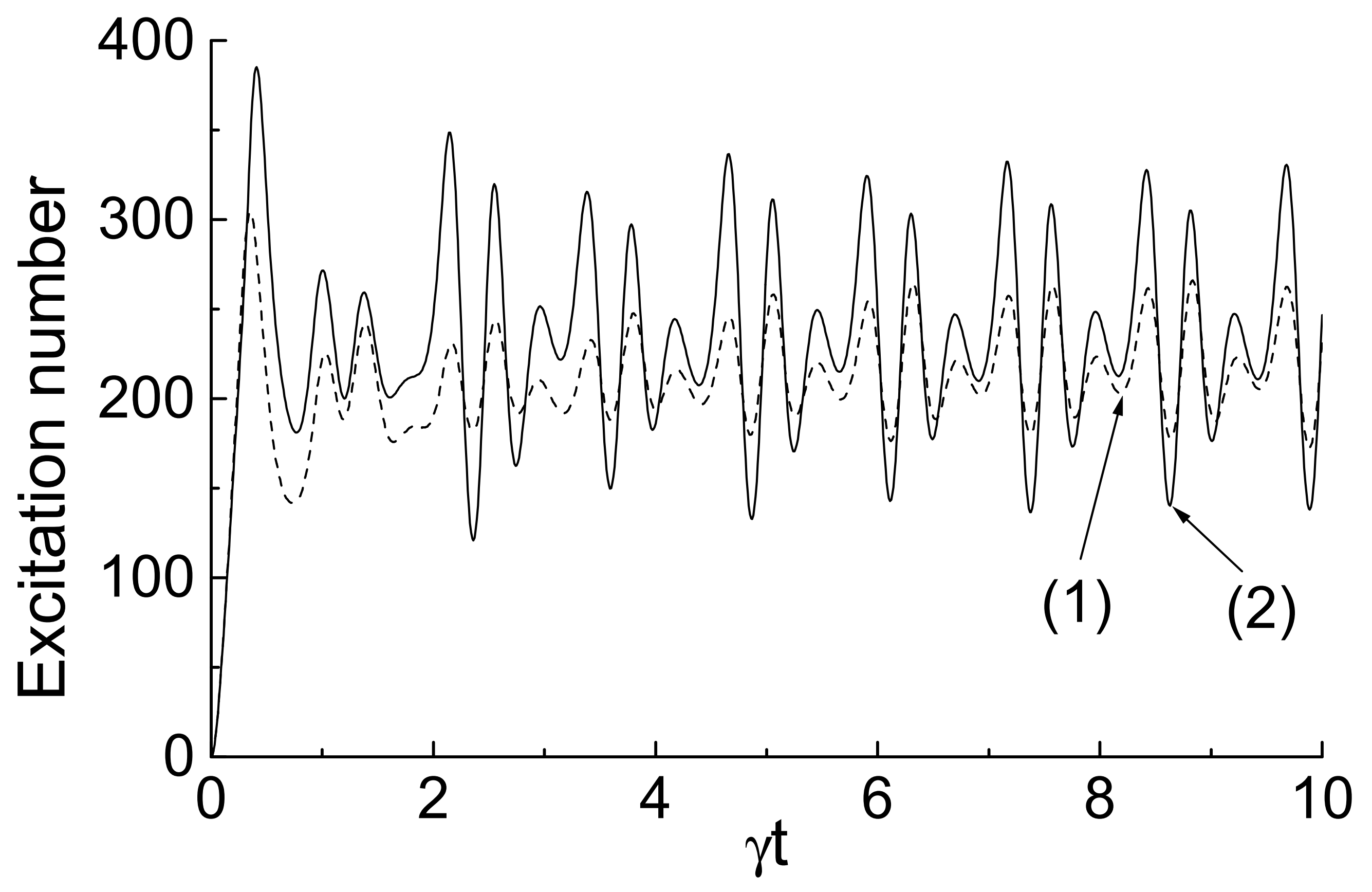




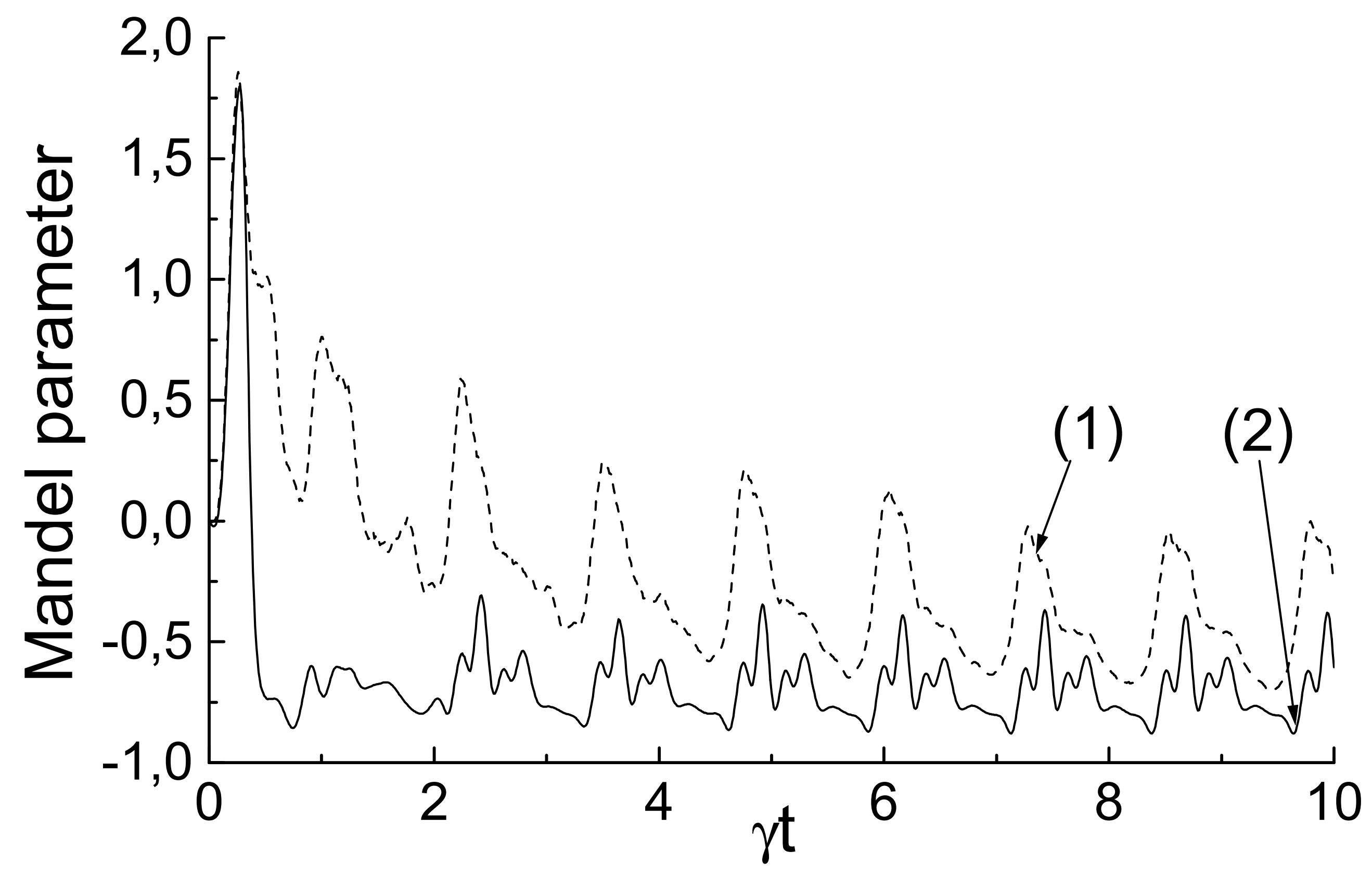




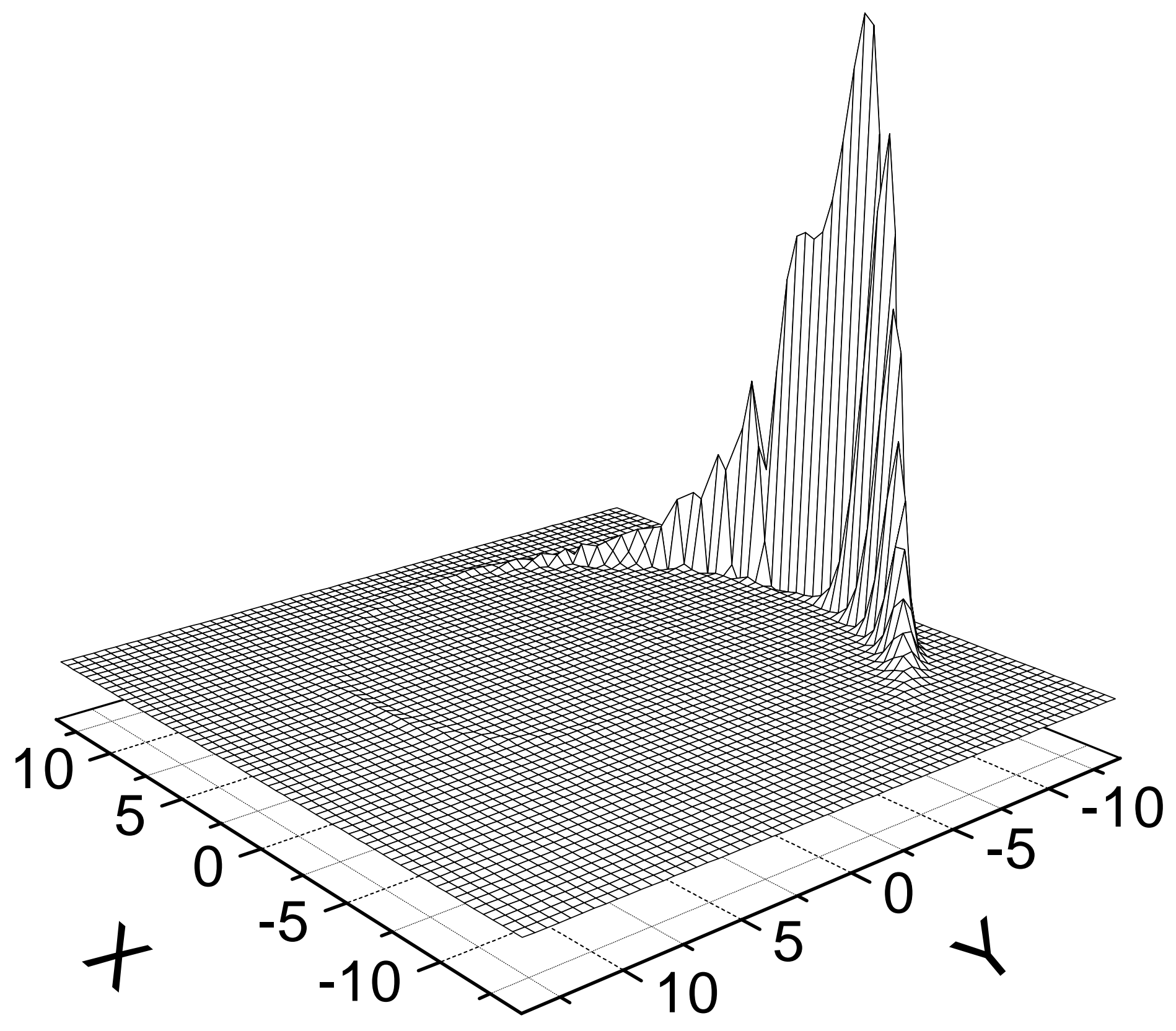




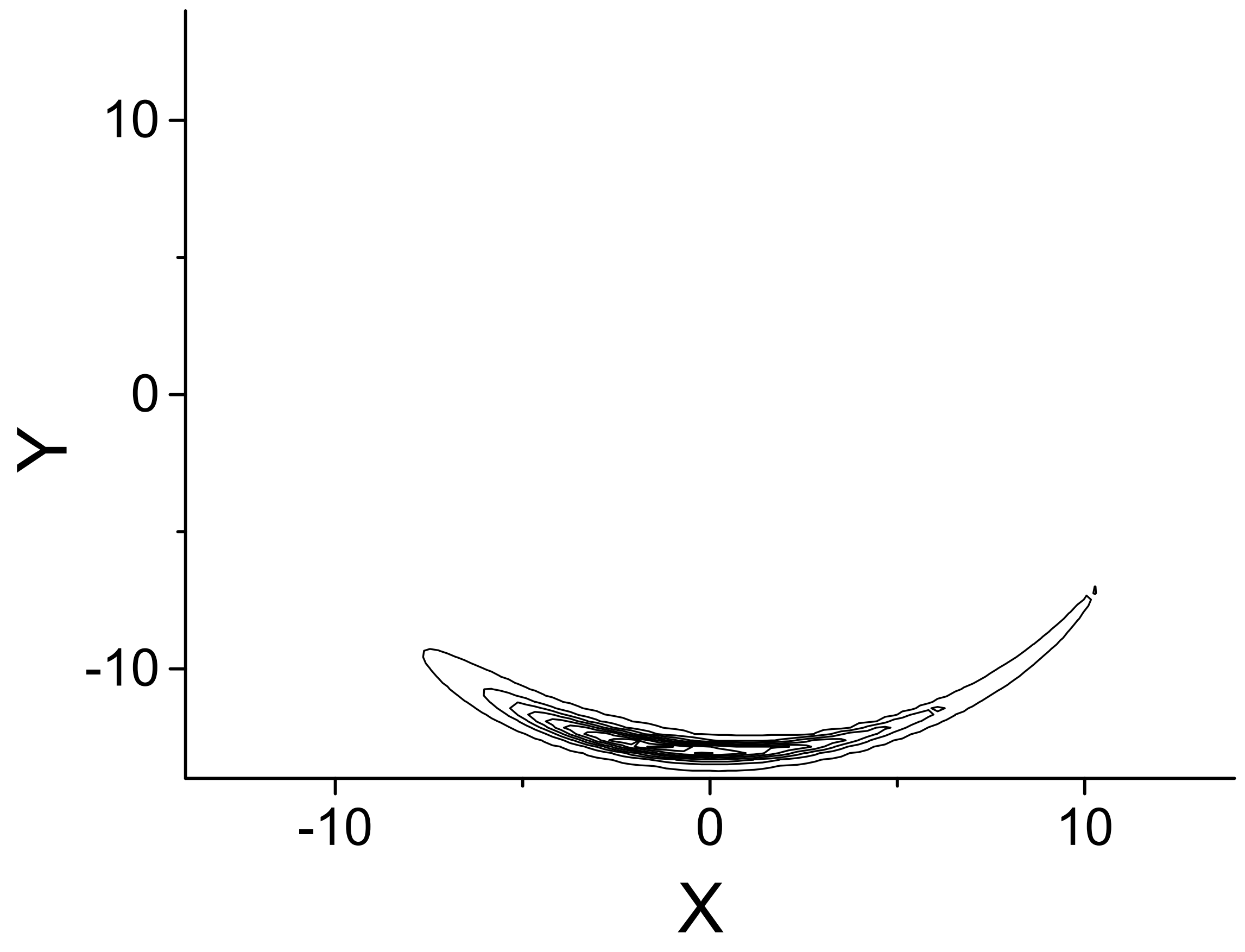




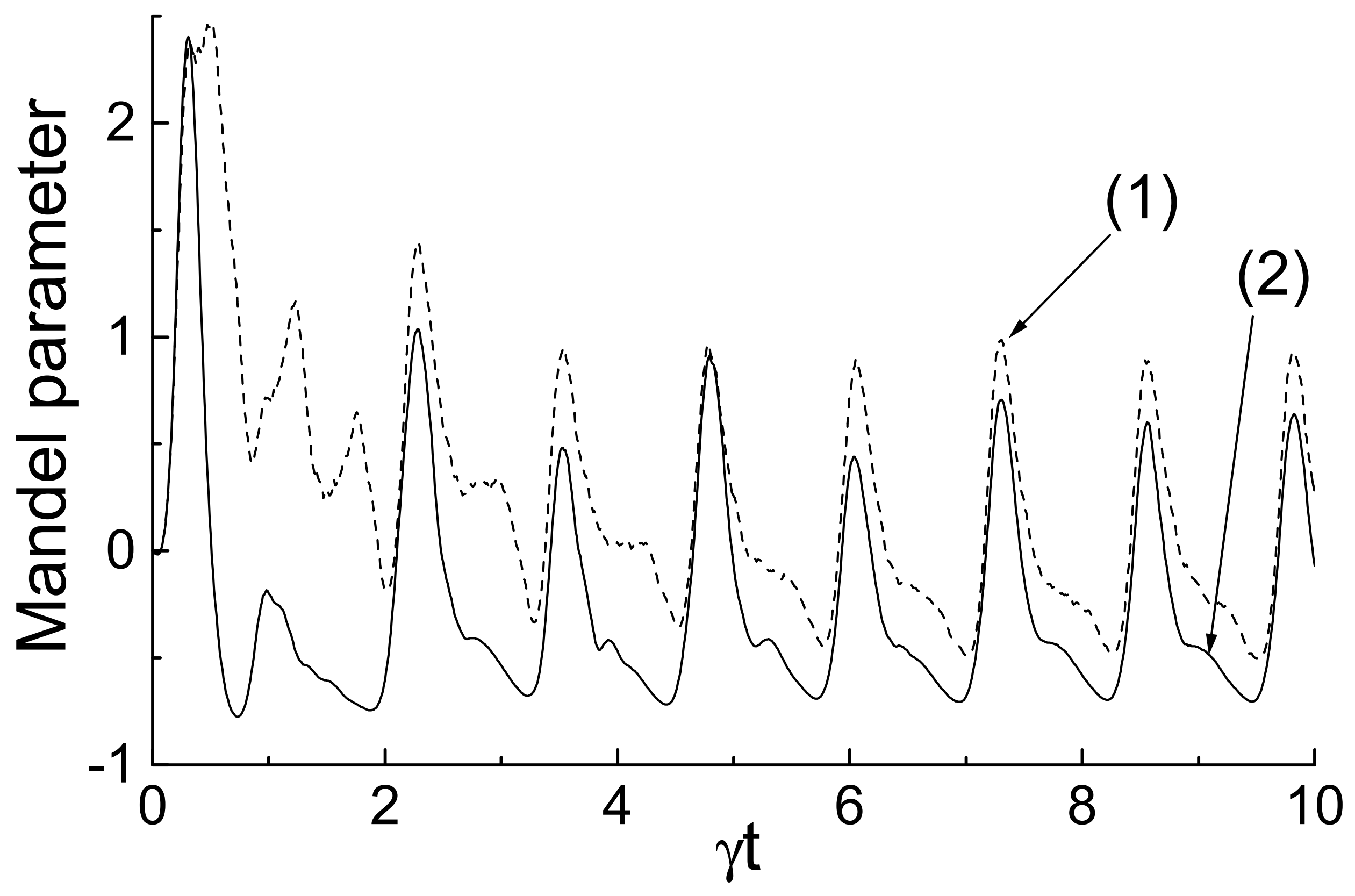




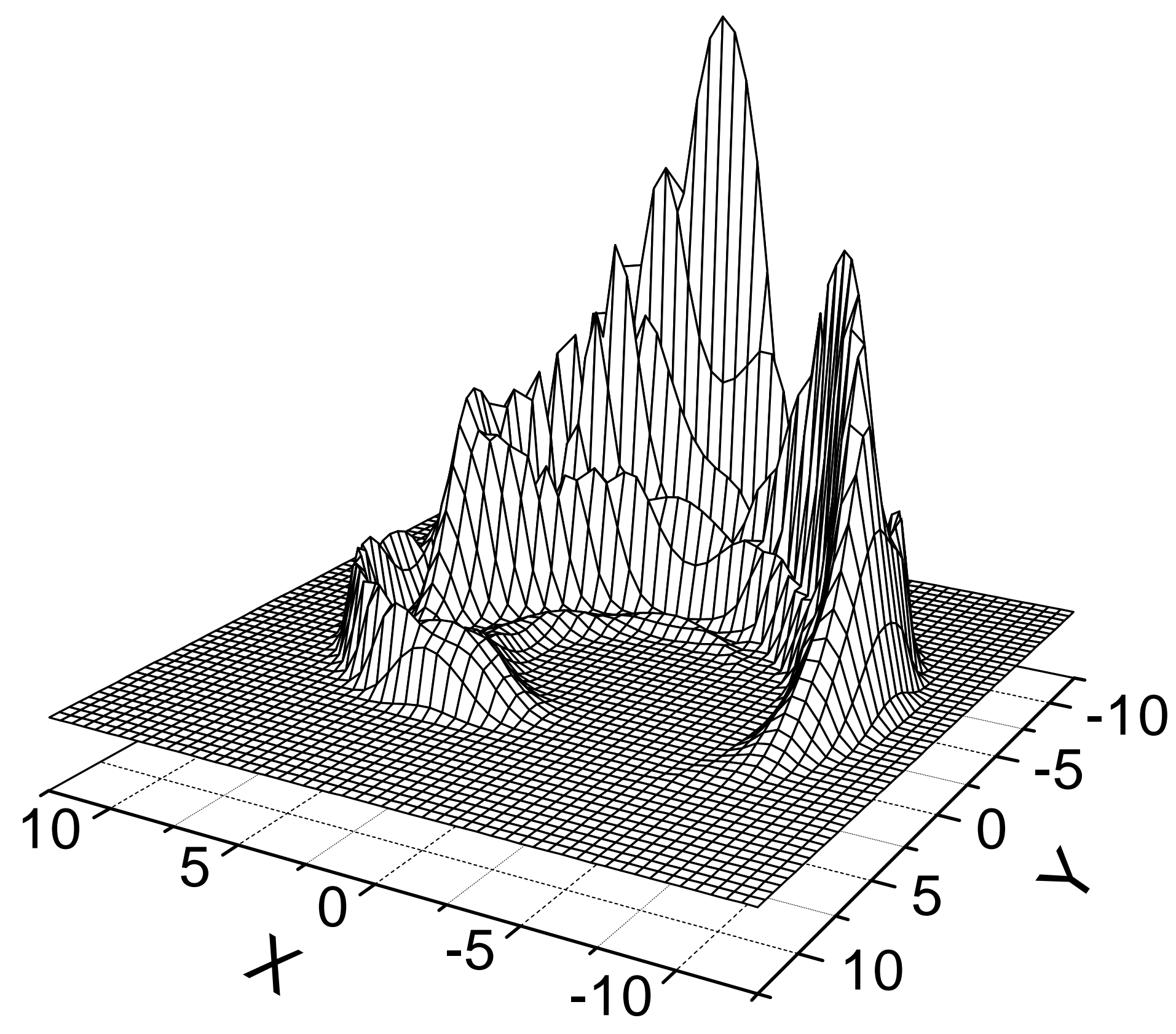




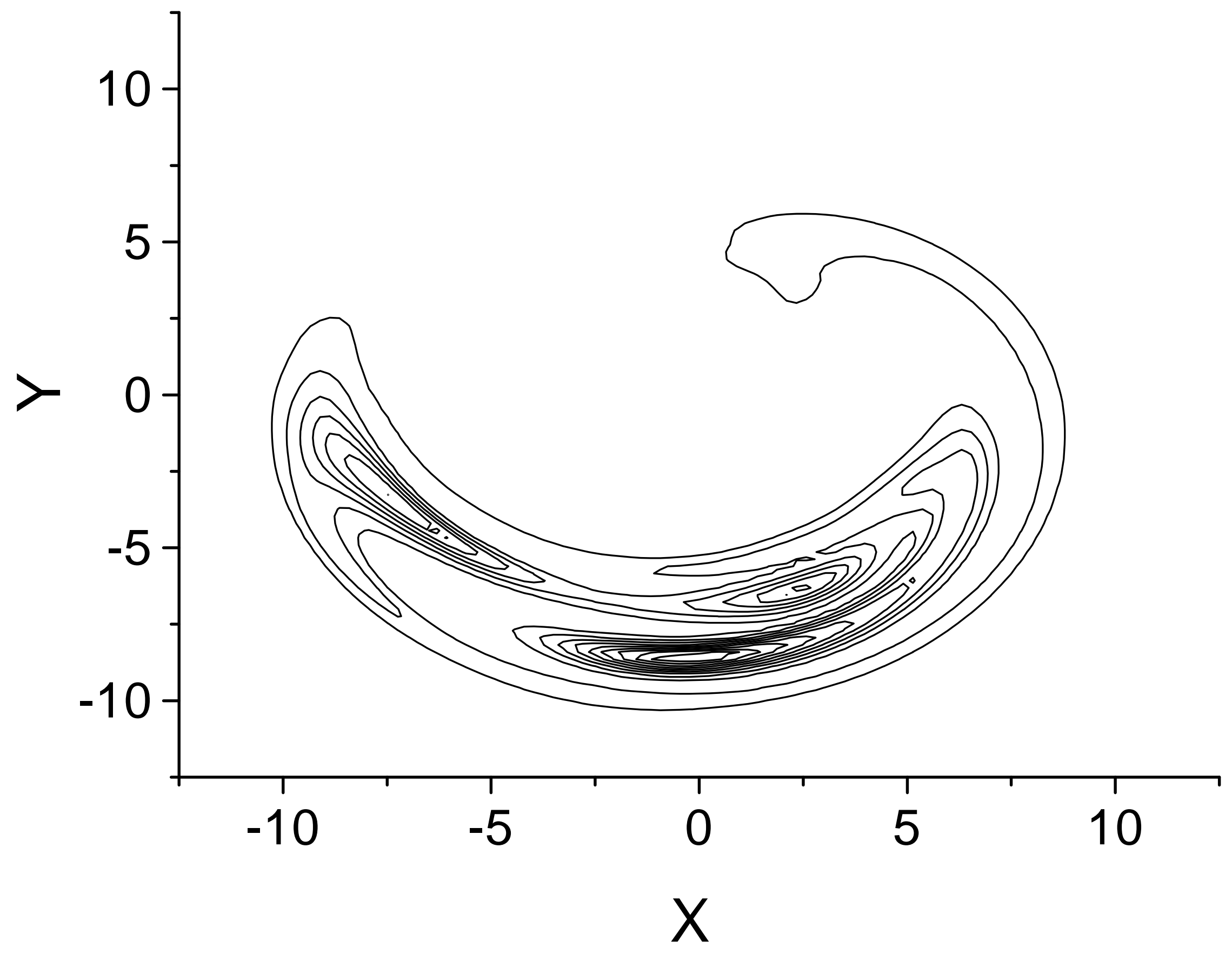




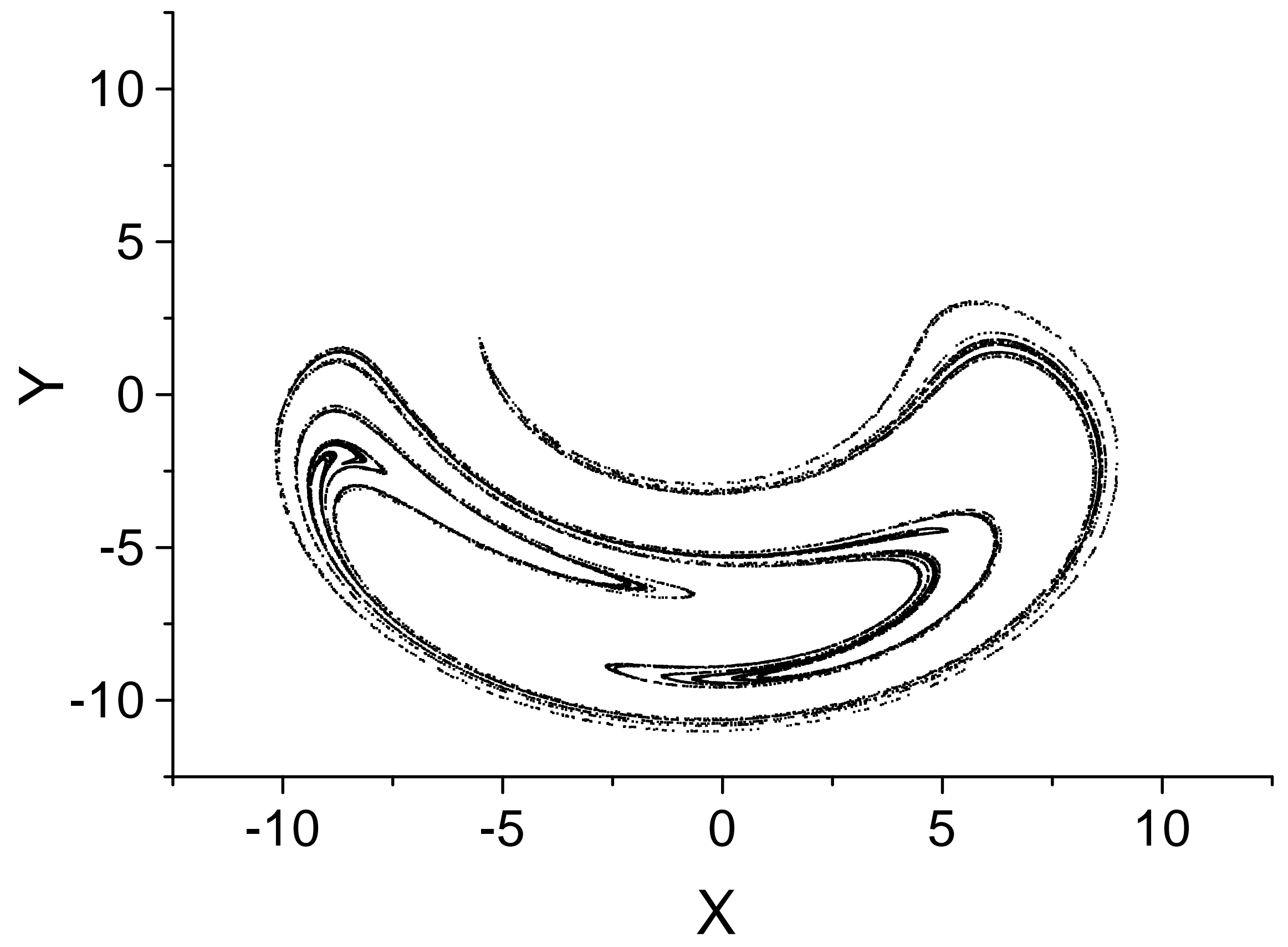




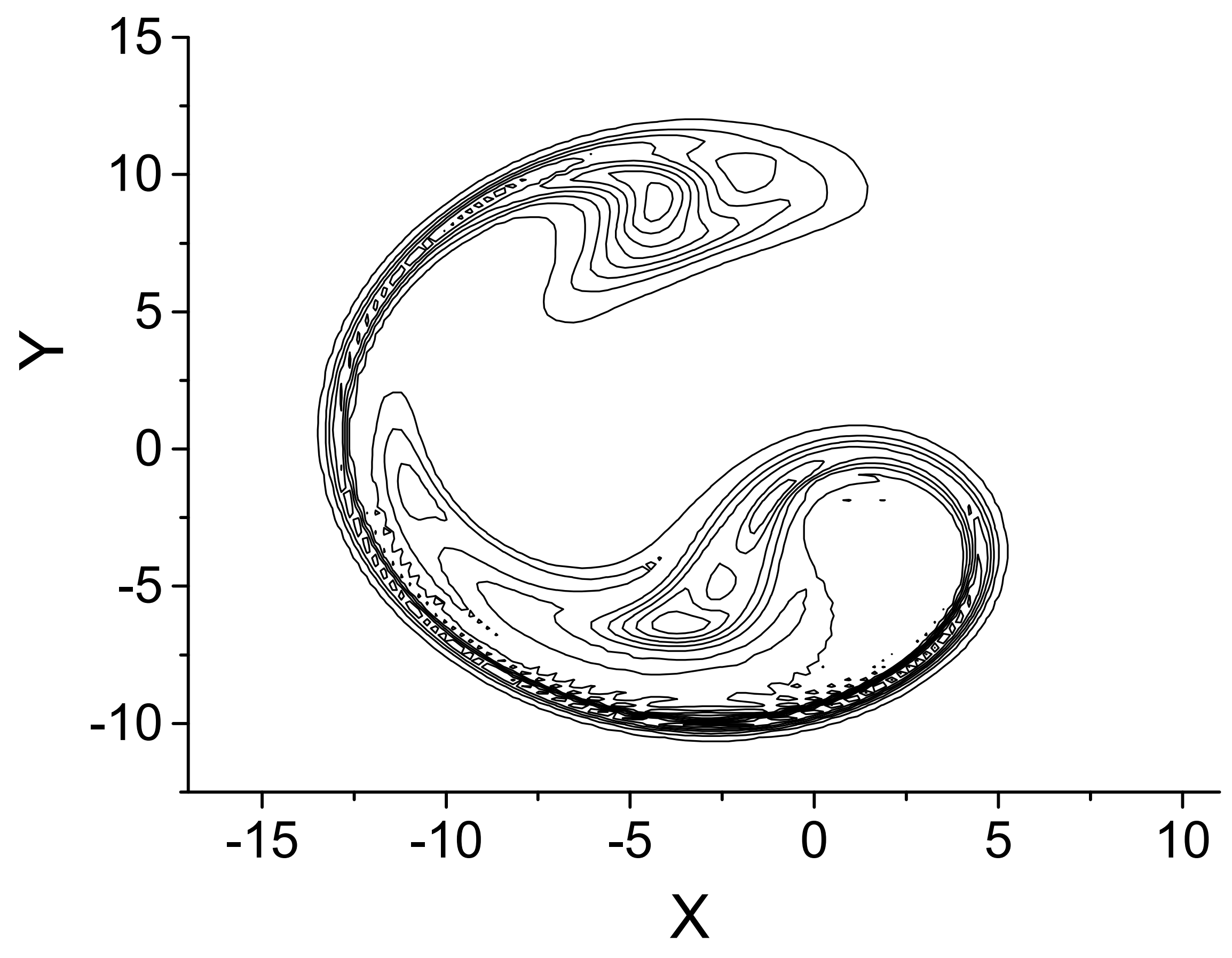




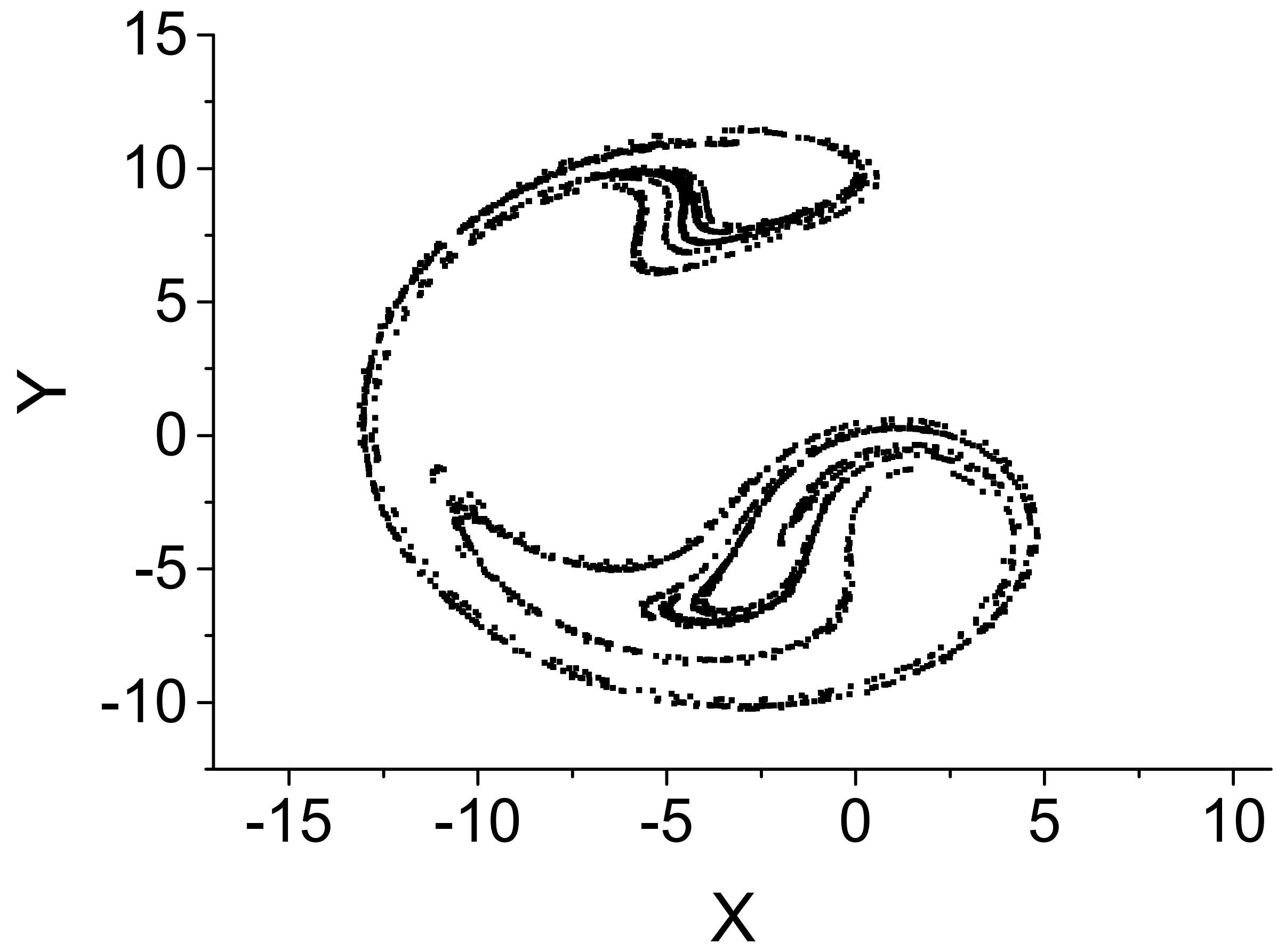




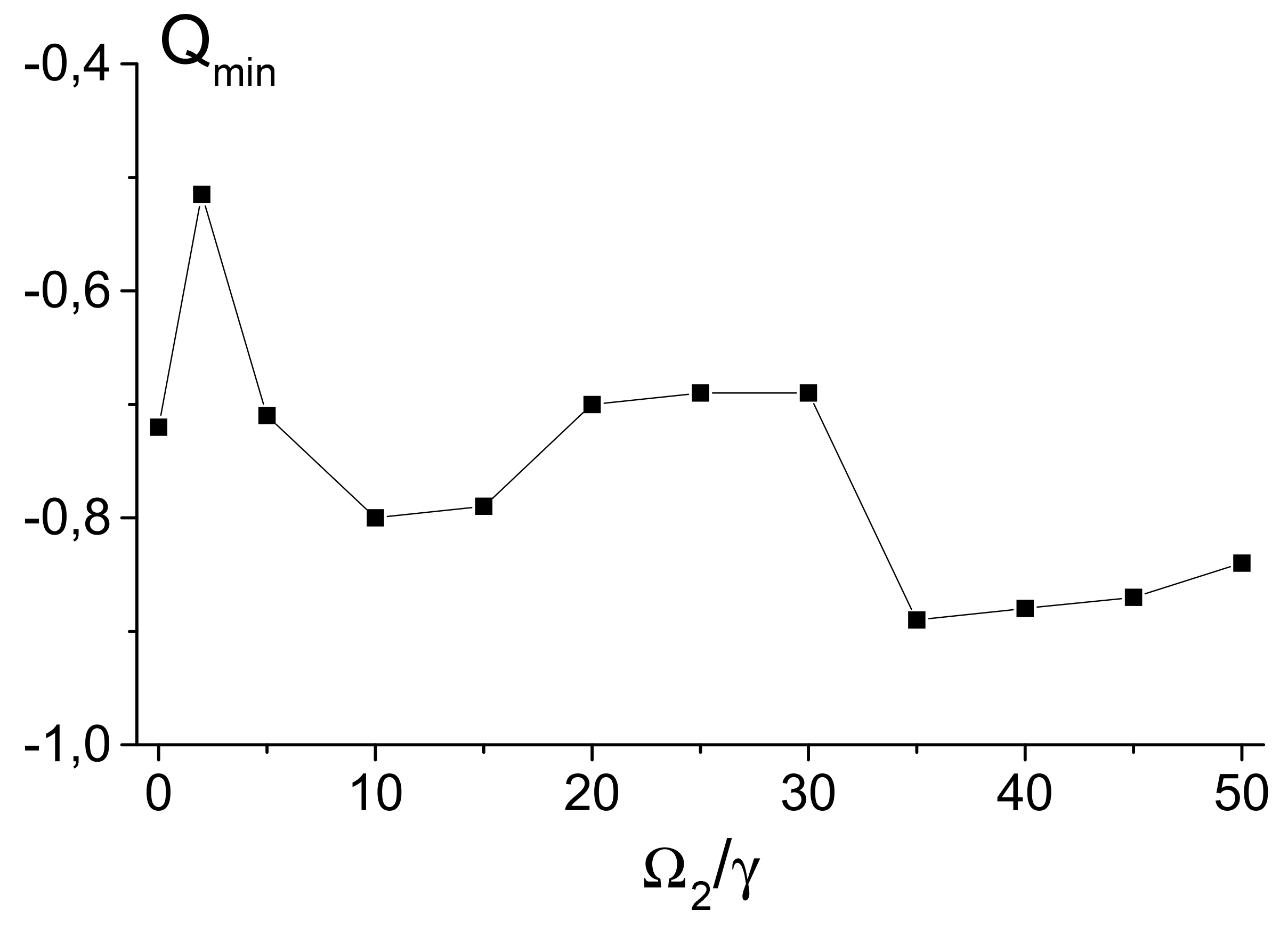




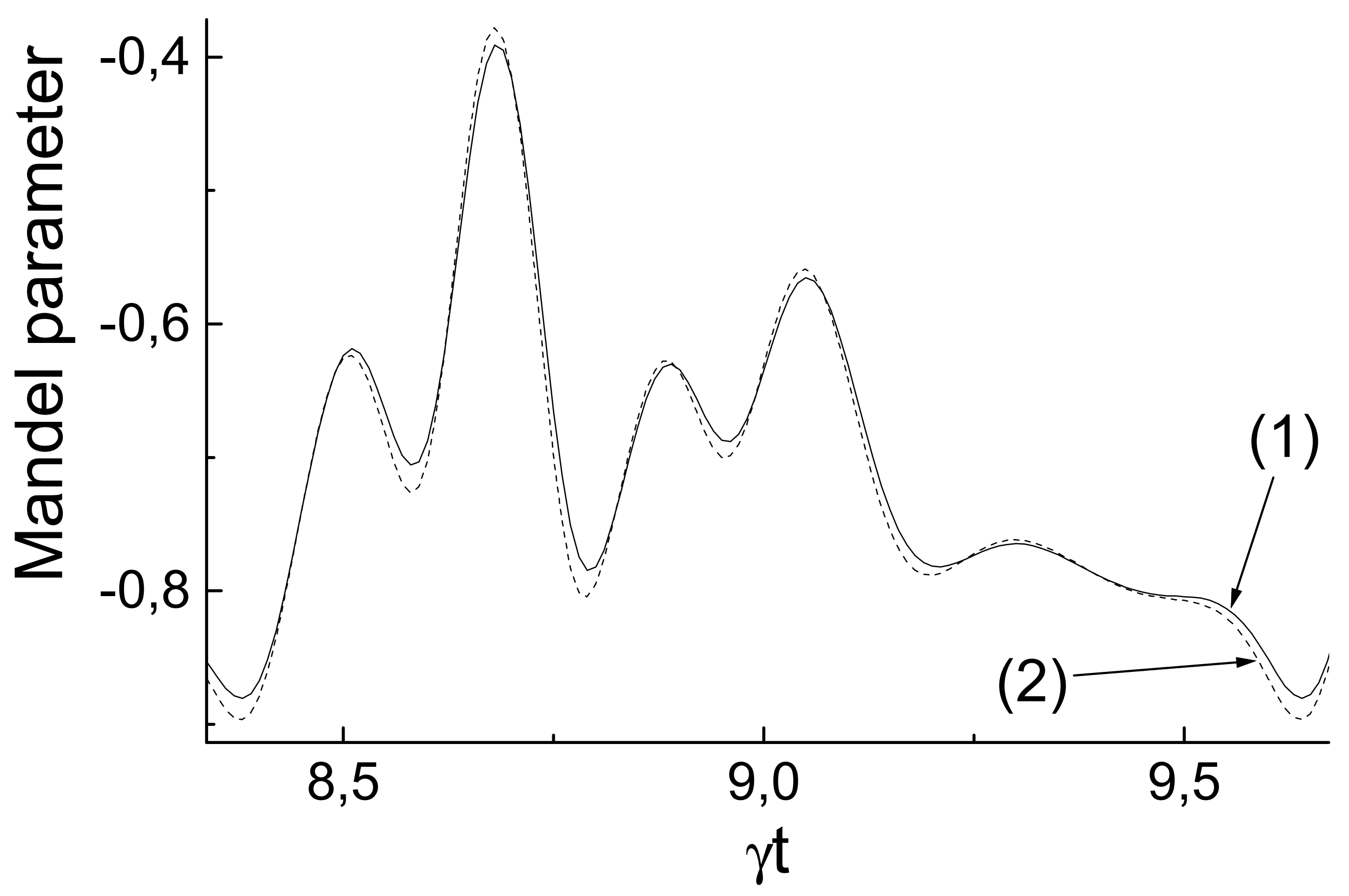




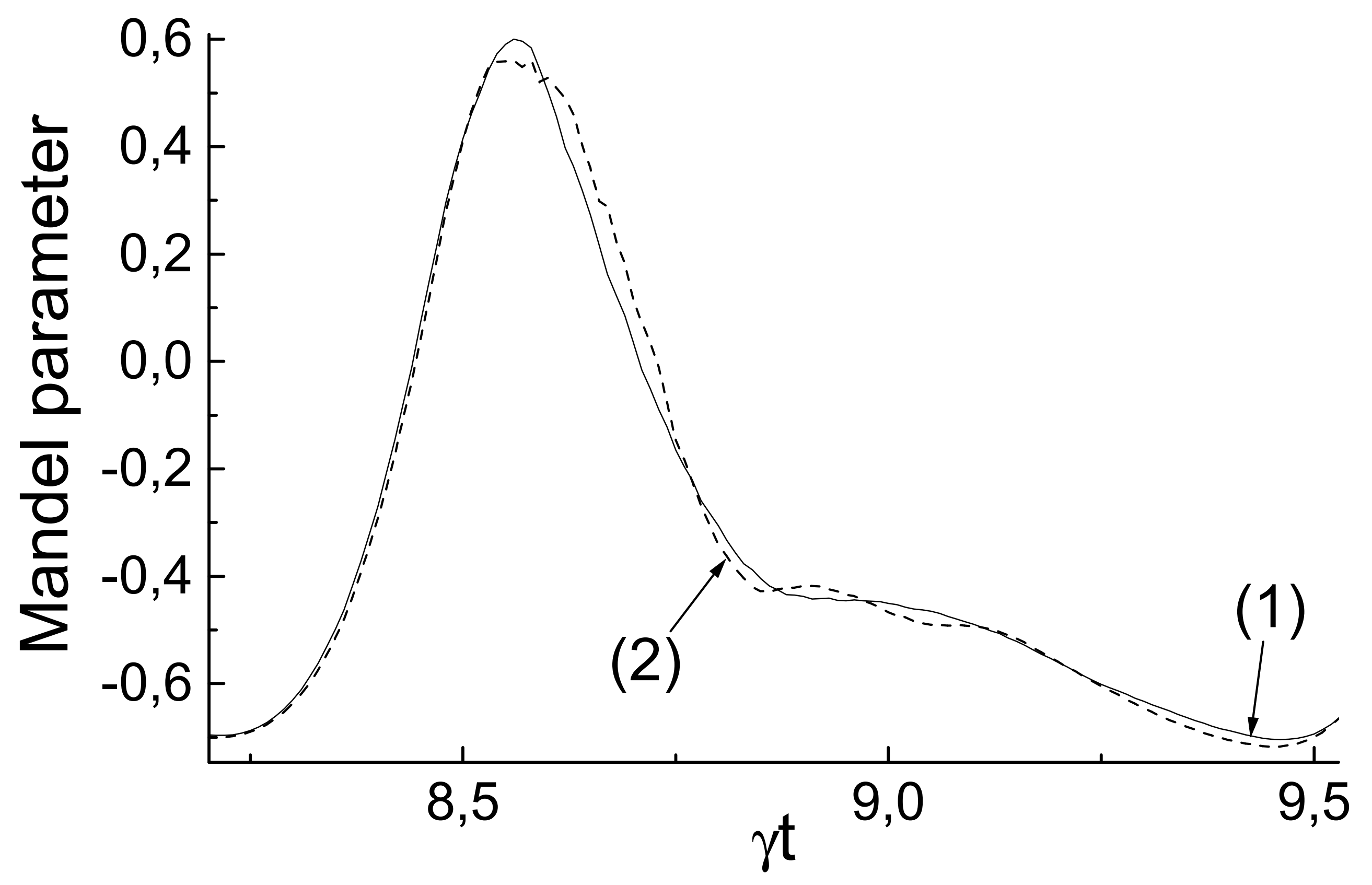




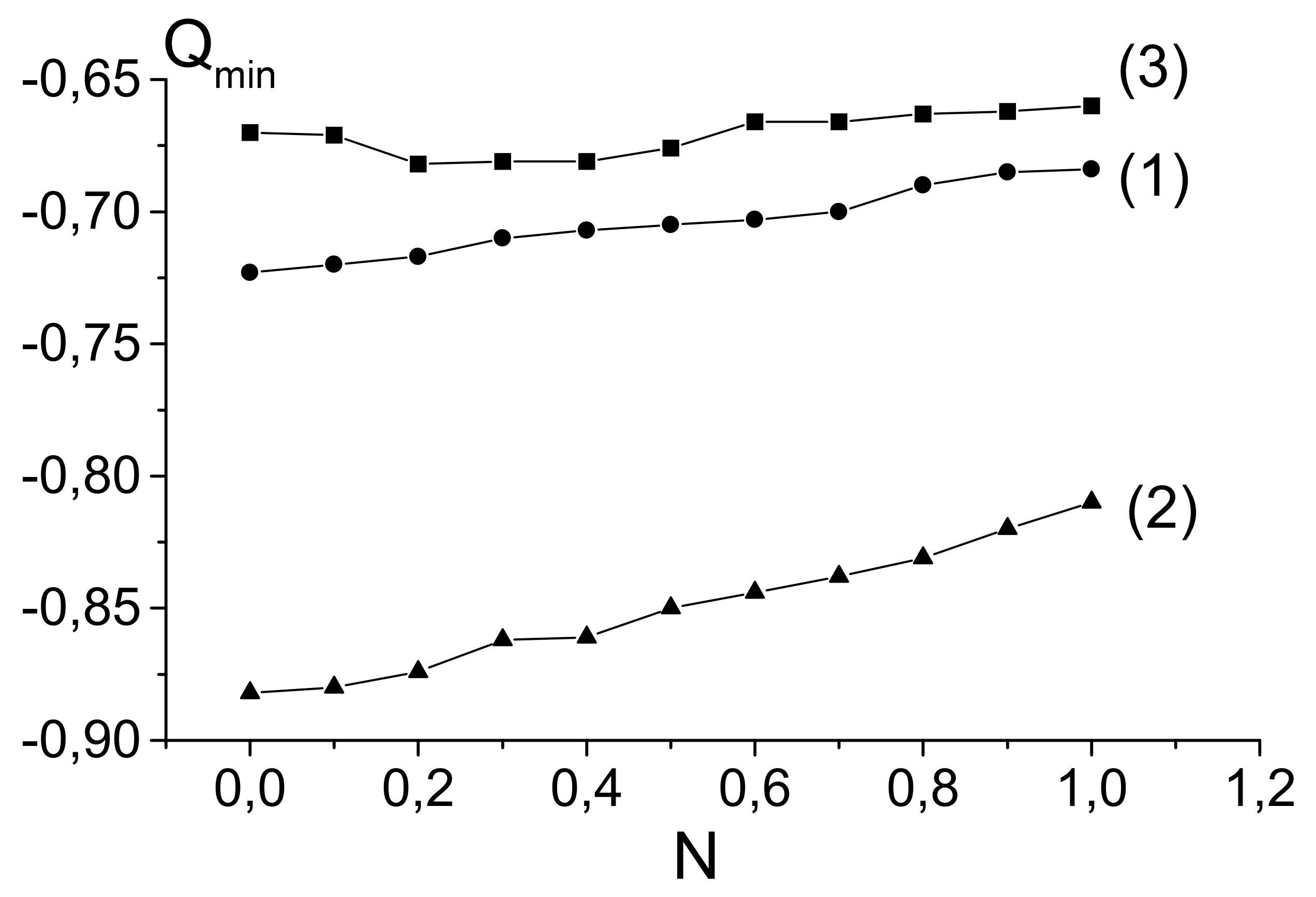

